\title{
Adaptive laboratory evolution resolves energy depletion to maintain high aromatic metabolite phenotypes in Escherichia coli strains lacking the Phosphotransferase System
}

McCloskey, Douglas; Xu, Sibei; Sandberg, Troy E.; Brunk, Elizabeth; Hefner, Ying; Szubin, Richard; Feist, Adam M.; Palsson, Bernhard O.

Published in:

Metabolic Engineering

Link to article, DOI:

10.1016/j.ymben.2018.06.005

Publication date:

2018

Document Version

Peer reviewed version

Link back to DTU Orbit

Citation (APA):

McCloskey, D., Xu, S., Sandberg, T. E., Brunk, E., Hefner, Y., Szubin, R., Feist, A. M., \& Palsson, B. O. (2018). Adaptive laboratory evolution resolves energy depletion to maintain high aromatic metabolite phenotypes in Escherichia coli strains lacking the Phosphotransferase System. Metabolic Engineering, 48, 233-242. https://doi.org/10.1016/j.ymben.2018.06.005

\section{General rights}

Copyright and moral rights for the publications made accessible in the public portal are retained by the authors and/or other copyright owners and it is a condition of accessing publications that users recognise and abide by the legal requirements associated with these rights.

- Users may download and print one copy of any publication from the public portal for the purpose of private study or research.

- You may not further distribute the material or use it for any profit-making activity or commercial gain

- You may freely distribute the URL identifying the publication in the public portal 


\section{Author's Accepted Manuscript}

Adaptive laboratory evolution resolves energy depletion to maintain high aromatic metabolite phenotypes in Escherichia coli strains lacking the Phosphotransferase System

Douglas McCloskey, Sibei Xu, Troy E. Sandberg, Elizabeth Brunk, Ying Hefner, Richard Szubin, Adam M. Feist, Bernhard O. Palsson

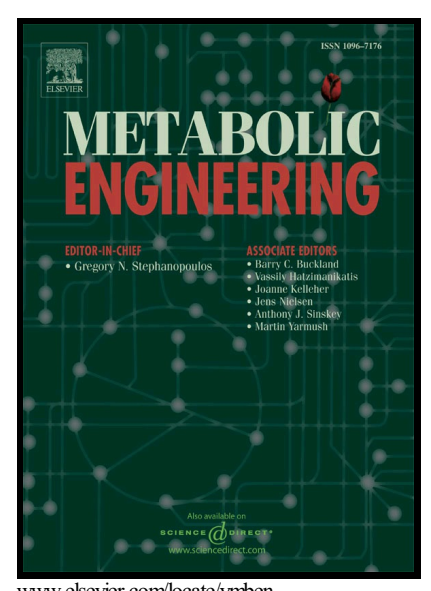

PII: $\quad$ S1096-7176(18)30017-X

DOI: $\quad$ https://doi.org/10.1016/j.ymben.2018.06.005

Reference: YMBEN1425

To appear in: Metabolic Engineering

Received date: 17 January 2018

Revised date: 8 June 2018

Accepted date: 9 June 2018

Cite this article as: Douglas McCloskey, Sibei Xu, Troy E. Sandberg, Elizabeth Brunk, Ying Hefner, Richard Szubin, Adam M. Feist and Bernhard O. Palsson, Adaptive laboratory evolution resolves energy depletion to maintain high aromatic metabolite phenotypes in Escherichia coli strains lacking the Phosphotransferase System, Metabolic Engineering, https://doi.org/10.1016/j.ymben.2018.06.005

This is a PDF file of an unedited manuscript that has been accepted for publication. As a service to our customers we are providing this early version of the manuscript. The manuscript will undergo copyediting, typesetting, and review of the resulting galley proof before it is published in its final citable form. Please note that during the production process errors may be discovered which could affect the content, and all legal disclaimers that apply to the journal pertain. 
Adaptive laboratory evolution resolves energy depletion to maintain high aromatic metabolite phenotypes in Escherichia coli strains lacking the Phosphotransferase System

Douglas McCloskey ${ }^{1,2}$, Sibei Xu${ }^{1}$, Troy E. Sandberg ${ }^{1}$, Elizabeth Brunk ${ }^{1}$, Ying Hefner ${ }^{1}$, Richard Szubin $^{1}$, Adam M. Feist ${ }^{1,2}$, and Bernhard O. Palsson ${ }^{1,2, *}$

${ }^{1}$ Department of Bioengineering, University of California - San Diego, La Jolla, CA 92093, USA.

${ }^{2}$ Novo Nordisk Foundation Center for Biosustainability, Technical University of Denmark, 2800 Lyngby, Denmark.

${ }^{*}$ Corresponding author, Department of Bioengineering, University of California, San Diego, 9500 Gilman Drive, La Jolla, CA 92093-0412, USA. Tel.: [858-534-5668]; Fax: [858-822-3120]; Email: palsson@ucsd.edu

\section{Abstract}

Aromatic metabolites provide the backbone for numerous industrial and pharmaceutical compounds of high value. The Phosphotransferase System (PTS) is common to many bacteria, and is the primary mechanism for glucose uptake by Escherichia coli. The PTS was removed to conserve phosphoenolpyruvate (pep), which is a precursor for aromatic metabolites and consumed by the PTS, for aromatic metabolite production. Replicate adaptive laboratory evolution (ALE) of PTS and detailed omics data sets collected revealed that the PTS bridged the gap between respiration and fermentation, leading to distinct high fermentative and high respiratory rate phenotypes. It was also found that while all strains retained high levels of aromatic amino acid (AAA) biosynthetic precursors, only one replicate from the high glycolytic clade retained high levels of intracellular AAAs. The fast growth and high AAA precursor 
phenotypes could provide a starting host for cell factories targeting the overproduction aromatic metabolites.

\section{Subject terms}

Adaptive laboratory evolution, mutation analysis, multi-omics analysis, systems biology, E. coli, ptsH, ptsl, and crr gene knockouts

\section{Introduction}

Aromatic metabolites provide the backbone for numerous nutraceuticals, pharmaceuticals, solvents, polymers, and a plethora of other commodity and fine chemical products ${ }^{1-6}$. Aromatic metabolites are derived from the shikimate pathway where glycolytic and pentose phosphate pathway (PPP) intermediates phosphoenolpyruvate (pep) and erythrose 4-phosphate (e4p) are combined and aromatized to chorismate (chor). Chor forms the building blocks for numerous aromatic compounds including the three aromatic amino acids (AAAs) L-tyrosine (tyr-L), Lphenylalanine (phe-L), and L-tryptophan (trp-L). Decades of research has focused on engineering cell factories for increased biosynthesis of AAAs and their intermediates in the hopes of developing a high aromatic compound producing strain from renewable resources ${ }^{1-29}$.

The primary route of glucose import in E. coli is via the Phosphotransferase System (PTS) ${ }^{7,10,13}$. The PTS is shared by many bacteria ${ }^{30}$. The PTS imports and phosphorylates carbon sources (such as glucose) while converting Phosphoenolpyruvate (pep) to Pyruvate (pyr), thus consuming one of two moles (i.e., 50\%) of pep produced by glycolysis. Strategies to conserve phosphoenolpyruvate for aromatic compound production include removal of $p t s H$, $p t s l$, and $\mathrm{crr}$ genes to disable the PTS. This forces the cell to utilize ATP-dependent glucose import 
mechanisms, which causes an energy drain leading to slow growth ${ }^{1,8}$. Engineering strategies are thus required to not only improve glucose utilization, but do so while conserving ATP. While strategies have been found, more optimal strategies, and a deeper understanding of the mechanisms of successful strategies would be greatly beneficial.

Instead of brute force and combinatorial optimization of genetic interventions that is typical of most metabolic engineering workflows, this study employed an alternative strategy whereby the PTS was removed from a pre-optimized E. coli strain, and evolution was allowed to select for solutions to improve the glucose import and growth phenotype of the ptsHIcrr strain. Novel solutions were found that allowed for both fast growth and high intracellular AAAs as well as their precursors. Key was the regeneration of energy in the absence of carbon source activation by pep. This lead to distinct high glycolytic and high respiratory rate phenotypes. In addition to the generation of beneficial strains for biotechnology applications, the in depth multiomics characterization of this study brings together biochemical details on a highly valued phenotype that can be utilized for further optimization.

\section{Results}

\section{ptsH, ptsl, and crr KOs and ALE}

A wild-type E. coli K-12 MG1655 strain previously evolved under glucose minimal media at $37^{\circ} \mathrm{C}$ ${ }^{31}$ (denoted as "Ref') was selected in order to minimize biological changes caused by adaption to the loss of a gene product from those caused by adaption to the growth conditions of the experiment. GLCptspp (genes ptsH, ptsl, and crr corresponding to enzymes HPr, El, and EIIA, respectively) were removed from Ref to generate strain uPtsHIcrr (denoted "unevolved ptsHIcrr 
knockout strain"). The loss of ptsH, ptsl, and crr disabled the PTS and resulted in a 79\% loss in fitness (Fig. 1B). Four uPtsHIcrr independently inoculated starting cultures were simultaneously evolved on glucose minimal media at $37^{\circ} \mathrm{C}$ in an automated ALE platform ${ }^{31,32}$ denoted "evolved ptsHlcrr knockout strains" or "ePtsHlcrr". A statistically significant increase in final fitness (Student's t-test, pvalue<0.05) was found in all ALE endpoints of the ePtsHlcrr lineages (ave \pm stdev $259 \pm 74 \%$ increase in fitness) compared to uPtsHlcrr.

\section{Conservation of pep initially translated to high aromatic amino}

\section{acid levels}

Inactivation of the PTS increased the availability of phosphoenolpyruvate (pep) for aromatic amino acid (AAA) biosynthesis by dissociating glucose import from pep consumption (Fig. 2A,B) 1,5. LC-MS/MS ${ }^{33,34}$ was used to quantify the levels of pep and AAAs. The levels of pep in uPtsHlcrr were 853.6\% greater than Ref (Fig. 2C, Fig. S1, Table S3). High intracellular pep translated to high levels of AAA pathway intermediates (Fig. S1) as well as the AAAs L-tyrosine (tyr-L), L-phenylalanine (phe-L), and L-tryptophan (trp-L), which were 99.9, 90.5, and 49.1\% greater in uPtsHlcrr than in Ref, respectively. Synthesis of the AAAs, is a linear pathway starting from erythrose 4-phosphate (e4p) and pep to the AAA intermediate chorismate (chor) where it then branches out to each of the three AAAs (Fig. S2). The loss of the PTS system thus imbalanced AAA levels as a result of the high level of pep that could not be converted to pyruvate in the unevolved KOs strain.

Adaptive evolution led to partial abrogation of this imbalance. High intracellular levels of AAAs were lost in all ePtsHlcrr strains except for phe-L and tyr-L in ePtsHlcrrO3 (Fig. 2C,Table S3). Levels of phe- $L$ waned to a $51.9 \%$ increase over Ref, while levels of tyr- $L$ shot up to a $626.6 \%$ increase over Ref. These results raised two questions: first, what were the mechanisms by 
which the ePtsHlcrr strains were able to recover growth and glucose import? And second, why was the high AAA phenotype lost in all strains except for ePtsHlcrr03? To answer these questions, DNA resequencing, RNA sequencing, fluxomics, and further metabolomics data were collected and analyzed for all strains.

\section{Loss of the PTS inactivated carbon catabolite repression (CCR)}

\section{via cAMP}

Evidence for strong activation of carbon catabolite repressed operons by CAMP-CRP was revealed by transcriptional profiling. Alternative glucose import and catabolism systems including glucokinase, the D-galactose symporter, and D-galactose $A B C$ transporter were upregulated in uPtsHIcrr compared to Ref (Fig. 3C, Table S4). In particular, genes encoding the galactitol and D-sorbital PTS were upregulated in uPtsHIcrr compared to Ref. These genes are positively regulated primarily by CRP-CAMP due to an insertion of IS3E in the gatR gene ${ }^{35}$, and serve as a proxy for CAMP-CRP activation status.

CRP activity is modulated by intracellular levels of its activator, CAMP. Highly elevated levels of cAMP were found in uPtsHIcrr compared to Ref (Fig. 4C, Table S3). The increased levels of CAMP and the activation of CAMP-CRP positively regulated genes indicated that a rise in intracellular cAMP levels in uPtsHIcrr inactivated CCR while in the presence of high levels of glucose. This observation is non-intuitive because phosphorylated EllA (encoded by crr) stimulates Adenylate Cyclase (AC, encoded by cyaA) to convert ATP to CAMP ${ }^{36}$ (Fig. $3 A$ ). The uPtsHIcrr strain lacks $c r r$. In addition, cAMP-CRP represses cyaA expression ${ }^{37}$, which was found to lead to a significant decrease in $c y a A$ expression in uPtsHIcrr. Previous studies have hypothesized that low levels of cAMP should be found in ptsHIcrr- strains ${ }^{14,38}$ due to decreased $A C$ activity and decreased $c y a A$ expression as highlighted above. By directly measuring cAMP, 
this study demonstrates that it can be produced at high levels in the absence of EIIA and at low cyaA expression levels due to the presence of an unknown activator ${ }^{39,40}$.

\section{Truncation of $A C$ altered expression of CCR associated genes}

Mutations in cyaA were found in ePtsHIcrr $01 / 03$ that appeared to silence the activation of cAMP-CRP positively regulated genes that were found in ePtsHIcrr (Fig. 4). Deletion (DEL) at the 456 residue in ePtsHlcrr01 and mobile element insertion (MOB) at the 518 residue in ePtsHIcrr03 truncated the peptide at the ElIA binding domain of AC (Fig. 4B). ePtsHIcrr01 and ePtsHIcrr03 had the lowest levels of cAMP compared to Ref, uPtsHIcrr, and the other ePtsHIcrr strains. This demonstrated that another effector was capable of stimulating AC activity at the EllA binding domain besides the gene product of $\mathrm{crr}{ }^{39,40}$, and that a physiological advantage could be had by removing the ability of that effector to bind AC.

The reduced levels of cAMP in ePtsHlcrr01/03 resulted in down regulation of many CCR operons. These included genes encoding the D-galactose ABC transporter, galactitol and Dsorbital PTS, and D-ribose symporter (Fig. 4C). However, genes encoding the D-galactose symporter and D-galactose catabolism remained significantly elevated in ePtsHIcrr01/03 compared to Ref. This indicated that passive import and catabolism of glucose via D-galactose symporters paired with increased ATP production from overflow metabolism ${ }^{41}$ (Fig. 1D) was sufficient to help restore glucose import capabilities in ePtsHIcrr01/03 strains ${ }^{3,8}$. In contrast, mutations in ATP-dependent glucose importers were found in ePtsHIcrr02/04 strains (discussed below). 


\section{Loss of PTS shifted glucose import towards symporters}

Inactivation of the PTS required a shift to alternative importers in order to enable glucose import in uPtsHIcrr. Genome-scale MFA ${ }^{42,43}$ was used to measure the fluxes into and out of the pep node (Fig. 5). In Ref, $100 \%$ of flux per mol of glucose imported was phosphorylated by the PTS. In contrast, $100 \%$ of flux per mol of imported glucose was taken up by alternative glucose importers and then phosphorylated by hexokinase (HEX1) in uPtsHIcrr and ePtsHIcrr strains. Expression profiling revealed that the primary glucose importer in uPtsHIcrr was via the Dgalactose symporter, encoded by galP with subsequent phosphorylation by hexokinase, encoded by glk (Fig. 2A-B, Fig. 5C, Table S4). Previous studies have demonstrated that the Dgalactose symporter has affinity for glucose, and that upregulation of galP and glk significantly increase glucose import at the expense of ATP ${ }^{3,8}$.

\section{Mutations in alternative importers helped to restore glucose}

\section{import capacity}

Initial upregulation of galP and glk was insufficient to restore glucose import capacity in uPtsHIcrr relative to the ref strains (Fig. 1D). In contrast to ePtsHIcrr01/03 where mutations targeted CAMP-CRP regulation, as discussed above, mutations that directly targeted sugar transport and catabolism operons appeared in ePtsHlcrr02/04. Affected genes included galR (Fig. 6) and nagC (Fig. 7).

Mutations at the galP TF binding site and in galR in ePtsHlcrr02 and 04, respectively, altered regulation of the D-galactose symporter (galP) and D-galactose $A B C$ transporter ( $m g / B A C$ ) by inhibiting the ability of operon-specific regulators, GalS and GalR, to repress expression (Fig. 6). An SNP in galR occurred in the small molecule binding domain that appeared to diminish the ability of effectors to activate GalR (Fig. 6A). ePtsHlcrr04 had the highest expression of 
galETKM and mgIBAC compared to all other strains. An SNP in galP targeted the GalR and GalS TF binding sites that appeared to negate repression by GalR and GalS (Fig. 6B). ePtsHlcrr02 had the highest expression of galP compared to all other strains.

A mutation in nagC in ePtsHlcrr04 altered regulation nAcGam catabolic genes (nagBAC) and nAcGam PTS component EIIB-nAcGam (nagE) (Fig. 7). NagC is a TF that inhibits expression of nagBAC and nagE operons. nagE encodes the PTS-import component that takes in exogenous $\mathrm{N}$-acetylglucosamine (nAcGam) that is derived either from turnover of the cell wall or found in the environment at the expense of pep. Note that nAcGam was not supplemented in the culture medium. Enzymes encoded by nagA and nagB then break down nAcGam to acetate, ammonia, and D-fructose-6-phosphate. The mutation occurred away from the DNA binding site and may affect the ability of effectors to active NagC. ePtsHIcrr04 had the highest expression of nagBAC and nagE compared to all other strains.

\section{Non-PTS glucose import and phosphorylation caused an energy drain}

The use of alternative glucose importers shifted the phosphoryl donor of g6p from pep to ATP due to the reliance upon HEX1 to convert glucose to g6p (Fig. 2 and Fig. 5). Reliance upon ATP-dependent phosphorylation of glucose resulted in a reduction in ATP and an elevation of AMP amounting to a significant reduction in the energy charge in uPtsHIcrr (Fig. 2C, Table S3). The energy drain caused by decreased availability of energy equivalents contributed to a decreased growth rate in uPtsHIcrr ${ }^{3,22,44}$ (Fig. 1D).

The activity of HEX1 was directly addressed by two of the four ePtsHIcrr strains. An intergenic mutation in front of the glk transcription start site in ePtsHlcrr02/04 appeared to downregulate 
expression of $g l k$ (Table S8). glk was downregulated in ePtsHIcrr02/04 compared to uPtsHlcrr and ePtsHIcrr01/03, but remained upregulated compared to Ref (Table S4). The mutation appeared to tune the expression of $g / k$ in order to balance the consumption of ATP with sufficient generation of glucose 6-phosphate ${ }^{3}$.

\section{Cell division defects triggered the Rcs Two Component system}

In addition to slow growth, morphological changes were observed when sampling and cultivating uPtsHIcrr (data not shown). Expression profiling revealed extensive upregulation of $n / p /$ in uPtsHIcrr compared to Ref (Table S4). Overexpression of $n / p /$ has been shown to lead to morphological changes that are indicative of cell division defects ${ }^{45,46}$. In combination with small heat shock proteins IbpA and IbpB, NIpl interrupts nucleoid division and assembly of FtsZ at the septum ${ }^{46}$. FtsZ forms a ring at the future replication site, which is the start of the first processes of cell division ${ }^{47}$. Additionally, in combination with the periplasmic protease Prc, Nipl forms a proteolytic system that regulates peptidoglycan synthesis by degrading MepS. MepS is a murein endopeptidase that participates in expansion of the peptidoglycan by breaking peptidoglycan cross links to allow for meurin incorporation ${ }^{48,49}$. A decrease in MepS activity through overexpression of $\mathrm{n} / \mathrm{p} /$ would lead to defective peptidoglycan synthesis.

Expression profiling also revealed that peptidoglycan synthesis abnormalities were detected by the Rcs phosphorelay system in uPtsHIcrr. The Rcs phosphorelay system responds to outer member $(\mathrm{OM})$ and peptidoglycan-related stress ${ }^{50-53}$, and controls genes related to motility, biofilm formation, virulence, $\mathrm{pH}$, osmolarity, periplasmic quality control, cell division, and DNA replication ${ }^{53-55}$ (Fig. 8A, see caption for mechanistic details of the Rcs phosphorelay system). Major Rcs signalling components comprising $r \operatorname{cs} A, r \operatorname{cs} B, r \operatorname{cs} C$, and $r \operatorname{cs} D$ were all upregulated in uPtsHlcrr compared to Ref, and in particular, rcsA, which positively upregulates itself upon 
activation, was massively upregulated (Fig. 8D, Table S4). Overactivation of the Rcs system is lethal ${ }^{56}$.

\section{Synergistic mutations between yrfF and rcs genes deactivate the Rcs system}

Overactivation of the Rcs phosphorelay system was negated by synergistic mutations in yrfF and rcs genes in all ePtsHlcrr strains (Fig. 8). yrfF was mutated in all ePtsHIcrr strains (Fig. 8B, D). YrfF constitutively inhibits the autophosphorylation of RcsC and subsequent phosphorylation of RcsD by RcsC. YrfF inactivation allows RcsD to phosphorylate and activate RcsB and RcsA, which activate downstream target genes. Combinations of mutation in rcsA, rcsB, and rcsD were found in all ePtsHIcrr strains (Fig. 8B, C, D) that complemented the yrfF mutations that could have prevented activation of RcsB and RcsA.

\section{LexA-mediated SOS response duplicated ATP synthase genes}

The loss of ptsHIcrr genes also resulted in a lexA-mediated SOS response ${ }^{57}$ (Fig. 9A-B). The SOS response upregulated a plethora of DNA repair genes as well as genes known to increase the probability of introducing mutations into the genome (Fig. 9A-C, Table S4). Significantly upregulated genes in uPtsHIcrr included $\operatorname{rec} A$, lexA, $\operatorname{din} B$ encoding polymerase IV, ruvABC involved in DNA double stranded break repair, and several others (Fig. 9C, Table S4). Overexpression of $\operatorname{din} B$ has been shown previously to confer a mutator phenotype ${ }^{58,59}$. The peptides encoded by the ruvABC operons form an enzymatic complex called the "resolvasome", which function together to resolve Holliday junctions formed during the recombinational repair of damaged double strand DNA, and to rescue blocked DNA replication forks via replication fork reversal ${ }^{60,61}$. 
The lexA-mediated SOS response manifested in a large duplication that included the ATP synthase complex in ePtsHlcrr02/04 (Fig. 9D-F). The duplicated region included 139 genes, among which 31.7 and $20.9 \%$ were doubled in expression compared to uPtsHlcrr and ePtsHIcrr01/03, respectively. Significantly up-regulated genes in ePtsHIcrr02/04 compared to uPtsHlcrr included cyaA and all ATP synthase complex genes (Fig. 9F, Table S4). The increased gene dosage of ATP synthase complex genes most likely contributed to the significant increase in the energy charge that was found in ePtsHIcrr02/04.

\section{Energy balance shifted flux into and out of the pep node}

Non-intuitively, the loss of flux through the PTS significantly increased the levels of pyruvate in ePtsHlcrr01 and ePtsHIcrr03. pyr levels increased by 870.9 and $1029.2 \%$ in ePtsHIcrr01/03 over Ref. The largest consuming flux of pep shifted from the PTS to PYK in all uPtsHIcrr and ePtsHIcrr strains. $\quad 37.9,122,143,92.7,93.4,126 \%$ of flux per mol of glucose of pep was converted to pyr via PYK in Ref, uPtsHIcrr, and ePtsHIcrr strains, respectively (Fig. 5B). The increase in pyr also increased the levels of L-alanine (ala-L), which is produced from pyr in a single nitrogen transfer step via glutamate-pyruvate aminotransaminase encoded by ala $A$ or alaC ${ }^{62}$. ala-L levels increased by 310.8 and $375.5 \%$ in ePtsHlcrr01/03 over Ref. In addition, excess pyr was excreted as Lactate (Fig. 1D, Table S2). This in particular would have been advantageous to the ePtsHlcrr01/03 strains to help rebalance the ratios of NAD and NADH which were significantly shifted to the oxidized state compared to ePtsHIcrr02/04 strains (Table S3) due to a higher glycolytic flux (Table S6).

The second largest drain of pep shifted from PYK to PPC. 16.0, 55.2, 12.0, 55.5, 22.2, 24.4\% of flux per mol of glucose of pep was diverted into the TCA cycle through PPC in Ref, uPtsHIcrr, and ePtsHIcrr strains, respectively (Fig. 5B). Including the contributions from PPC, 
ePtsHlcrr02/04 strains had a significantly greater amount of flux through the TCA cycle. The increased flux through the TCA cycle would be advantageous in the ePtsHIcrr02/04 strains in particular for contributing additional redox potentials to the electron transport chain to complement the increased dosage of ATP synthase. In contrast, ePtsHlcrr01/03 strains had significantly less TCA cycle flux, but significant acetate excretion (Fig. 1D, Table S2). This would be advantageous in the ePtsHIcrr01/03 strains for regenerating ATP through Acetate Kinase.

\section{Conclusion}

In this study, a desirable phenotype for biotechnology applications (i.e., high intracellular AAA levels) was lost in all strains but one during ALE. Mutations were found that altered regulation of CCR and secondary glucose import and catabolism pathways in order to restore glucose uptake from the loss of the primary glucose import and catabolism route via the PTS. The energy drain caused by the use of secondary glucose import and catabolism pathways was offset by changes in flux through glycolysis, lactate and acetate secretion, TCA cycle usage, and a duplication of the ATP synthase complex. From these genetic and physiological adaptations emerged a high glycolytic clade (ePtsHlcrr01/03) and a high respiratory clade (ePtsHlcrr02/04). Only the ePtsHlcrr03 strain from the high glycolytic clade retained high levels of intracellular AAAs, however all strains retained high levels of AAA biosynthetic precursors. 


\section{Acknowledgements}

We thank José Utrilla for helpful discussion and guidance when implementing the knockouts in the pre-evolved strain. We thank Jamey Young for helpful discussions throughout the MFA analysis. We thank Laurence Yang for helpful discussions regarding optimization and statistical analysis. This work was funded by the Novo Nordisk Foundation Grant Number NNF10CC1016517.

\section{Contributions}

D.M. designed the experiments; generated the strains; conducted all aspects of the metabolomics, fluxomics, phenomics, transcriptomics, and genomics experiments; performed all multi-omics statistical, graph, and modeling analyses; and wrote the manuscript. T.E.S. ran the ALE experiments. E.B. assisted with structural analysis. R.S. processed the DNA and RNA samples. S.X. assisted with metabolomics and fluxomics data collection, sample processing, and peak integration. Y.H. assisted with fluxomics data collection and sample processing. A.M.F designed and supervised the evolution experiments, and contributed to the data analysis and the manuscript. B.O.P conceived and outlined the study, supervised the data analysis, and co-wrote the manuscript. 


\section{Competing financial interests}

The authors declare no competing financial interests.

\section{References}

1. Flores, N., Xiao, J., Berry, A., Bolivar, F. \& Valle, F. Pathway engineering for the production of aromatic compounds in Escherichia coli. Nat. Biotechnol. 14, 620-623 (1996).

2. Escalante, A. et al. Metabolic engineering for the production of shikimic acid in an evolved Escherichia coli strain lacking the phosphoenolpyruvate: carbohydrate phosphotransferase system. Microb. Cell Fact. 9, 21 (2010).

3. Balderas-Hernández, V. E. et al. Metabolic engineering for improving anthranilate synthesis from glucose in Escherichia coli. Microb. Cell Fact. 8, 19 (2009).

4. Vargas-Tah, A. et al. Production of cinnamic and p-hydroxycinnamic acid from sugar mixtures with engineered Escherichia coli. Microb. Cell Fact. 14, 6 (2015).

5. Chávez-Béjar, M. I., Báez-Viveros, J. L., Martínez, A., Bolívar, F. \& Gosset, G. Biotechnological production of I-tyrosine and derived compounds. Process Biochem. 47, $1017-1026(2012 / 7)$.

6. Chávez-Béjar, M. I. et al. Metabolic engineering of Escherichia coli to optimize melanin synthesis from glucose. Microb. Cell Fact. 12, 108 (2013).

7. Flores, S., Gosset, G., Flores, N., de Graaf, A. A. \& Bolívar, F. Analysis of carbon metabolism in Escherichia coli strains with an inactive phosphotransferase system by (13)C labeling and NMR spectroscopy. Metab. Eng. 4, 124-137 (2002).

8. Hernández-Montalvo, V. et al. Expression of galP and glk in a Escherichia coli PTS mutant 
restores glucose transport and increases glycolytic flux to fermentation products. Biotechnol. Bioeng. 83, 687-694 (2003).

9. Flores, N. et al. Role of pyruvate oxidase in Escherichia coli strains lacking the phosphoenolpyruvate:carbohydrate phosphotransferase system. J. Mol. Microbiol. Biotechnol. 8, 209-221 (2004).

10. Flores, N. et al. Adaptation for fast growth on glucose by differential expression of central carbon metabolism and gal regulon genes in an Escherichia coli strain lacking the phosphoenolpyruvate:carbohydrate phosphotransferase system. Metab. Eng. 7, 70-87 (2005).

11. Flores, N. et al. Growth recovery on glucose under aerobic conditions of an Escherichia coli strain carrying a phosphoenolpyruvate:carbohydrate phosphotransferase system deletion by inactivating arcA and overexpressing the genes coding for glucokinase and galactose permease. J. Mol. Microbiol. Biotechnol. 13, 105-116 (2007).

12. Lévy, S., Zeng, G. Q. \& Danchin, A. Cyclic AMP synthesis in Escherichia coli strains bearing known deletions in the pts phosphotransferase operon. Gene 86, 27-33 (1990).

13. Olvera, L. et al. Transcription analysis of central metabolism genes in Escherichia coli. Possible roles of sigma38 in their expression, as a response to carbon limitation. PLoS One 4, e7466 (2009).

14. Sigala, J. C. et al. Acetate metabolism in Escherichia coli strains lacking phosphoenolpyruvate: carbohydrate phosphotransferase system; evidence of carbon recycling strategies and futile cycles. J. Mol. Microbiol. Biotechnol. 16, 224-235 (2009).

15. Báez, J. L., Bolívar, F. \& Gosset, G. Determination of 3-deoxy-D-arabino-heptulosonate 7phosphate productivity and yield from glucose in Escherichia coli devoid of the glucose phosphotransferase transport system. Biotechnol. Bioeng. 73, 530-535 (2001).

16. Martínez, K. et al. Coutilization of glucose and glycerol enhances the production of aromatic compounds in an Escherichia coli strain lacking the phosphoenolpyruvate: carbohydrate 
phosphotransferase system. Microb. Cell Fact. 7, 1 (2008).

17. Escalante, A., Salinas Cervantes, A., Gosset, G. \& Bolívar, F. Current knowledge of the Escherichia coli phosphoenolpyruvate-carbohydrate phosphotransferase system: peculiarities of regulation and impact on growth and product formation. Appl. Microbiol. Biotechnol. 94, 1483-1494 (2012).

18. Flores, N. et al. New insights into the role of sigma factor RpoS as revealed in escherichia coli strains lacking the phosphoenolpyruvate:carbohydrate phosphotransferase system. J. Mol. Microbiol. Biotechnol. 14, 176-192 (2008).

19. Aguilar, C. et al. Genetic changes during a laboratory adaptive evolution process that allowed fast growth in glucose to an Escherichia coli strain lacking the major glucose transport system. BMC Genomics 13, 385 (2012).

20. Sabido, A. et al. Physiological and transcriptional characterization of Escherichia coli strains lacking interconversion of phosphoenolpyruvate and pyruvate when glucose and acetate are coutilized. Biotechnol. Bioeng. 111, 1150-1160 (2014).

21. Cortés-Tolalpa, L. et al. Global transcriptomic analysis of an engineered Escherichia coli strain lacking the phosphoenolpyruvate: carbohydrate phosphotransferase system during shikimic acid production in rich culture medium. Microb. Cell Fact. 13, 28 (2014).

22. Fuentes, L. G. et al. Modification of glucose import capacity in Escherichia coli: physiologic consequences and utility for improving DNA vaccine production. Microb. Cell Fact. 12, 42 (2013).

23. Licona-Cassani, C. et al. Inactivation of pyruvate kinase or the phosphoenolpyruvate: sugar phosphotransferase system increases shikimic and dehydroshikimic acid yields from glucose in Bacillus subtilis. J. Mol. Microbiol. Biotechnol. 24, 37-45 (2014).

24. Muñoz, A. J. et al. Metabolic engineering of Escherichia coli for improving L-3,4dihydroxyphenylalanine (L-DOPA) synthesis from glucose. J. Ind. Microbiol. Biotechnol. 38, 1845-1852 (2011). 
25. Balderas-Hernández, V. E., Hernández-Montalvo, V., Bolívar, F., Gosset, G. \& Martínez, A. Adaptive evolution of Escherichia coli inactivated in the phosphotransferase system operon improves co-utilization of xylose and glucose under anaerobic conditions. Appl. Biochem. Biotechnol. 163, 485-496 (2011).

26. Ikeda, M. Towards bacterial strains overproducing L-tryptophan and other aromatics by metabolic engineering. Appl. Microbiol. Biotechnol. 69, 615-626 (2006).

27. Berry, A. Improving production of aromatic compounds in Escherichia coli by metabolic engineering. Trends Biotechnol. 14, 250-256 (1996).

28. Ueguchi, C., Misonou, N. \& Mizuno, T. Negative control of rpoS expression by phosphoenolpyruvate: carbohydrate phosphotransferase system in Escherichia coli. J. Bacteriol. 183, 520-527 (2001).

29. Flores, S. et al. Nutrient-scavenging stress response in an Escherichia coli strain lacking the phosphoenolpyruvate: carbohydrate phosphotransferase system, as explored by gene expression profile analysis. J. Mol. Microbiol. Biotechnol. 10, 51-63 (2005).

30. Erni, B. The bacterial phosphoenolpyruvate: sugar phosphotransferase system (PTS): an interface between energy and signal transduction. J. Iran. Chem. Soc. 10, 593-630 (2013).

31. LaCroix, R. A. et al. Use of Adaptive Laboratory Evolution To Discover Key Mutations Enabling Rapid Growth of Escherichia coli K-12 MG1655 on Glucose Minimal Medium. Appl. Environ. Microbiol. 81, 17-30 (2015).

32. Sandberg, T. E. et al. Evolution of Escherichia coli to $42^{\circ} \mathrm{C}$ and subsequent genetic engineering reveals adaptive mechanisms and novel mutations. Mol. Biol. Evol. 31, 26472662 (2014).

33. McCloskey, D., Gangoiti, J. A., Palsson, B. O. \& Feist, A. M. A pH and solvent optimized reverse-phase ion-paring-LC-MS/MS method that leverages multiple scan-types for targeted absolute quantification of intracellular metabolites. Metabolomics 11, 1338-1350 (2015). 
34. McCloskey, D., Utrilla, J., Naviaux, R. K., Palsson, B. O. \& Feist, A. M. Fast Swinnex filtration (FSF): a fast and robust sampling and extraction method suitable for metabolomics analysis of cultures grown in complex media. Metabolomics 11, 198-209 (2014).

35. Nobelmann, B. \& Lengeler, J. W. Molecular analysis of the gat genes from Escherichia coli and of their roles in galactitol transport and metabolism. J. Bacteriol. 178, 6790-6795 (1996).

36. Pastan, I. \& Perlman, R. Cyclic adenosine monophosphate in bacteria. Science 169, 339344 (1970).

37. Aiba, H. Transcription of the Escherichia coli adenylate cyclase gene is negatively regulated by cAMP-cAMP receptor protein. J. Biol. Chem. 260, 3063-3070 (1985).

38. Krin, E., Sismeiro, O., Danchin, A. \& Bertin, P. N. The regulation of Enzyme IIA(Glc) expression controls adenylate cyclase activity in Escherichia coli. Microbiology 148, 15531559 (2002).

39. Park, Y.-H., Lee, B. R., Seok, Y.-J. \& Peterkofsky, A. In vitro reconstitution of catabolite repression in Escherichia coli. J. Biol. Chem. 281, 6448-6454 (2006).

40. Reddy, P. \& Kamireddi, M. Modulation of Escherichia coli adenylyl cyclase activity by catalytic-site mutants of protein IIA(Glc) of the phosphoenolpyruvate:sugar phosphotransferase system. J. Bacteriol. 180, 732-736 (1998).

41. Basan, M. et al. Overflow metabolism in Escherichia coli results from efficient proteome allocation. Nature 528, 99-104 (2015).

42. McCloskey, D., Young, J. D., Xu, S., Palsson, B. O. \& Feist, A. M. MID Max: LC-MS/MS Method for Measuring the Precursor and Product Mass Isotopomer Distributions of Metabolic Intermediates and Cofactors for Metabolic Flux Analysis Applications. Anal. Chem. 88, 1362-1370 (2016).

43. McCloskey, D., Young, J. D., Xu, S., Palsson, B. O. \& Feist, A. M. Modeling Method for Increased Precision and Scope of Directly Measurable Fluxes at a Genome-Scale. Anal. 
Chem. 88, 3844-3852 (2016).

44. Valgepea, K., Adamberg, K. \& Vilu, R. Decrease of energy spilling in Escherichia coli continuous cultures with rising specific growth rate and carbon wasting. BMC Syst. Biol. 5, 106 (2011).

45. Ohara, M., Wu, H. C., Sankaran, K. \& Rick, P. D. Identification and characterization of a new lipoprotein, NIpl, in Escherichia coli K-12. J. Bacteriol. 181, 4318-4325 (1999).

46. Tao, J. et al. Heat shock proteins IbpA and IbpB are required for NIpl-participated cell division in Escherichia coli. Front. Microbiol. 6, 51 (2015).

47. Bi, E. F. \& Lutkenhaus, J. FtsZ ring structure associated with division in Escherichia coli. Nature 354, 161-164 (1991).

48. Singh, S. K., SaiSree, L., Amrutha, R. N. \& Reddy, M. Three redundant murein endopeptidases catalyse an essential cleavage step in peptidoglycan synthesis of Escherichia coli K12. Mol. Microbiol. 86, 1036-1051 (2012).

49. Singh, S. K., Parveen, S., SaiSree, L. \& Reddy, M. Regulated proteolysis of a cross-linkspecific peptidoglycan hydrolase contributes to bacterial morphogenesis. Proc. Natl. Acad. Sci. U. S. A. 112, 10956-10961 (2015).

50. Evans, K. L., Kannan, S., Li, G., de Pedro, M. A. \& Young, K. D. Eliminating a set of four penicillin binding proteins triggers the Rcs phosphorelay and Cpx stress responses in Escherichia coli. J. Bacteriol. 195, 4415-4424 (2013).

51. Farris, C., Sanowar, S., Bader, M. W., Pfuetzner, R. \& Miller, S. I. Antimicrobial peptides activate the Rcs regulon through the outer membrane lipoprotein RcsF. J. Bacteriol. 192, 4894-4903 (2010).

52. Laubacher, M. E. \& Ades, S. E. The Rcs phosphorelay is a cell envelope stress response activated by peptidoglycan stress and contributes to intrinsic antibiotic resistance. $J$. Bacteriol. 190, 2065-2074 (2008).

53. Majdalani, N. \& Gottesman, S. The Rcs phosphorelay: a complex signal transduction 
system. Annu. Rev. Microbiol. 59, 379-405 (2005).

54. Majdalani, N. \& Gottesman, S. Genetic dissection of signaling through the Rcs phosphorelay. Methods Enzymol. 423, 349-362 (2007).

55. Pannen, D., Fabisch, M., Gausling, L. \& Schnetz, K. Interaction of the RcsB Response Regulator with Auxiliary Transcription Regulators in Escherichia coli. J. Biol. Chem. 291, 2357-2370 (2016).

56. Cho, S.-H. et al. Detecting envelope stress by monitoring $\beta$-barrel assembly. Cell 159, 1652-1664 (2014).

57. Kreuzer, K. N. DNA damage responses in prokaryotes: regulating gene expression, modulating growth patterns, and manipulating replication forks. Cold Spring Harb. Perspect. Biol. 5, a012674 (2013).

58. Godoy, V. G., Jarosz, D. F., Walker, F. L., Simmons, L. A. \& Walker, G. C. Y-family DNA polymerases respond to DNA damage-independent inhibition of replication fork progression. EMBO J. 25, 868-879 (2006).

59. McKenzie, G. J., Lee, P. L., Lombardo, M. J., Hastings, P. J. \& Rosenberg, S. M. SOS mutator DNA polymerase IV functions in adaptive mutation and not adaptive amplification. Mol. Cell 7, 571-579 (2001).

60. Seigneur, M., Bidnenko, V., Ehrlich, S. D. \& Michel, B. RuvAB acts at arrested replication forks. Cell 95, 419-430 (1998).

61. Baharoglu, Z., Petranovic, M., Flores, M.-J. \& Michel, B. RuvAB is essential for replication forks reversal in certain replication mutants. EMBO J. 25, 596-604 (2006).

62. Kim, S. H., Schneider, B. L. \& Reitzer, L. Genetics and regulation of the major enzymes of alanine synthesis in Escherichia coli. J. Bacteriol. 192, 5304-5311 (2010).

63. Stülke, J. \& Hillen, W. Carbon catabolite repression in bacteria. Curr. Opin. Microbiol. 2, 195-201 (1999).

64. Franchini, A. G., Ihssen, J. \& Egli, T. Effect of Global Regulators RpoS and Cyclic- 
AMP/CRP on the Catabolome and Transcriptome of Escherichia coli K12 during Carbonand Energy-Limited Growth. PLoS One 10, e0133793 (2015).

65. Yong, H. T. et al. Development of a system for discovery of genetic interactions for essential genes in Escherichia coli K-12. Genes Genet. Syst. 88, 233-240 (2013).

66. Pristovšek, P. et al. Structural Analysis of the DNA-binding Domain of theErwinia amylovora RcsB Protein and Its Interaction with the RcsAB Box. J. Biol. Chem. 278, 17752-17759 (2003). 
Fig. 1 Evolution of knockout (KO) strains from a pre-evolved (i.e., optimized) wild-type strain. A) Wild-type (wt) E. coli (MG1655 K12) was previously evolved on glucose minimal media at $37^{\circ} \mathrm{C}^{31}$. An isolate from the endpoint of the evolutionary experiment was selected as the starting strain for subsequent $\mathrm{KO}$ of ptsHlcrr and adaptive laboratory evolution (ALE). B) Adaptive laboratory evolution trajectories of the evolved knockout lineages. -Omics data collected included metabolomics, fluxomics, physiology, DNA resequencing, and transcriptomics. C) The Glucose specific phosphotransferase system (GLCptspp) was disabled by the gene KO. GLCptspp is the primary importer of glucose into the cell, and converts glucose to glucose 6 phosphate (g6p) by phosphotransfer from Phosphoenolpyruvate (pep) to Pyruvate (pyr). D) Growth rate and uptake and secretion rates for unevolved KO (uPtsHlcrr) and evolved KOs (ePtsHlcrr). For comparison, The reference strain (Ref) had a growth rate of 0.92 (+/- 0.01) $\mathrm{hr}^{-1}$ and glucose uptake and acetate secretion rates of $12.0(+/-0.12), 8.18(+/-0.15) \mathrm{mmol}^{*} \mathrm{gDCW}^{-1 *} \mathrm{hr}^{-1}$, respectively. 
Fig. $2 \mathrm{KO}$ of the primary glucose importation system (ptsHlcrr) increased the availability of PEP for aromatic metabolite production, but caused a drain in available ATP through the upregulation of secondary glucose importation systems that utilize ATP instead of PEP. A) A network and mechanistic schematic of the PTS system. The metabolite conversions in red are removed through the ptsHlcrr KO. B) A schematic of passive/active glucose importers. C) Metabolite concentrations of the aromatic amino acids L-phenylalanine (phe-L), L-tryptophan (trp-L), and L-tyrosine (tyr-L), and their precursor phosphoenolpyruvate (pep) taken from 3 biological replicates analyzed in duplicate $(n=6)$. Also shown are the metabolite concentrations for L-histidine (his-L), which is derived from ribose, and energy charge ratio calculated as $\frac{a t p+\frac{a d p}{2}}{a t p+a d p+a m p}$. See Fig. S1 for levels of aromatic amino acid pathway intermediate levels. The box represent the interquartile ranges, the line through the box represents the median, and whiskers represent the maximum and minimum ranges. 
Fig. 3 Carbon catabolite repression (CCR) in the absence of crr. Carbon catabolite repression is a global regulatory mechanism that inhibits the expression of genes involved in the catabolism of secondary carbon sources in the presence of a preferred carbon source. A) Schematic of components and their interactions involved in CCR. During normal growth on glucose, EIIA, encoded by crr, interacts with EIIB, encoded by PtsG, by transferring a phosphate group to EIIB. Thus, during normal growth, EIIB is primarily phosphorylated while EIIA is primarily unphosphorylated. In the absence of glucose, ElIA remains primarily phosphorylated (See ${ }^{63}$ for a review). Phosphorylated EllA in the presence of an unidentified effector, positively regulates adenylate cyclase $(A C)$ activity ${ }^{39,40}$. Activation of $A C$ increases cAMP levels ${ }^{36}$. cAMP activates CRP, which triggers myriad transcriptional regulatory changes involved in CCR as well as other cellular processes ${ }^{64}$ B) Regulatory diagram of selected components involved in CCR. C) Shift in expression towards galactose symporter and ABC transporter, and glucokinase. gatYZABCD is regulated primarily by CRP-CAMP due to an insertion of IS3E in the gatR gene ${ }^{35}$. gatYZABCD serves as a reporter of CRP-CAMP activation.

Fig. 4 Mutations in cyaA that help restore basal levels of cAMP. A) Schematic of the cyaA operon and network diagram of cyaA. cyaA encodes Adenylate Cyclase (AC), which converts ATP to $\mathrm{CAMP}^{36}$. B) Crystal structure of $c y a A$. Deletion (DEL) at the 456 residue in ePtsHIcrr01 and mobile element insertion (MOB) at the 518 residue target the catalytic region of $\mathrm{AC}$. Both mutations truncate the peptide at the ElIA binding domain. Mutations are highlighted in red. The catalytic region is highlighted in purple. The crr interaction domain and truncated region are highlighted in pink. C) Mutation frequency and expression profiles of cyaA. Box and whiskers plot of CAMP metabolite concentrations from biological triplicates measured in analytical duplicate $(n=6)$. Besides the significantly different metabolite levels of cAMP between the Ref and uPtsHlcrr strain, ePtsHIcrr03 has significanlty less $(p<0.05)$ cAMP than ePtsHlcrr04. The box represent the interquartile ranges, the line through the box represents the median, and 
whiskers represent the maximum and minimum ranges. Given the decreased expression of cyaA in uPtsHIcrr, the location of the mutations that specifically cleaved cyaA at the $c r r$ binding domain, and the removal of $\mathrm{crr}$, it would appear that there is another mechanism by which $c y a A$ can be activated to produce cAMP.

\section{Fig. 5 Flux sum through Phosphoenolpyruvate (pep). A) Network diagram of the fluxes into and out of the pep node. B) Measured}

\section{absolute fluxes. C) Relative expression levels.}

Fig. 6 Mutations that alter regulation of the D-galactose symporter and D-galactose ABC transporter. Expression of the D-galactose symporter (galP) and D-galactose ABC transporter $(m g \mid B A C)$ are increased by mutations that target galP and galR. A) Crystal structure of GalR. The SNP in galR in ePtsHIcrr04 occurred in the small molecule binding domain. Mutations are highlighted in red. The small molecule binding domain is highlighted in cyan. The H-T-H DNAbinding region is highlighted in violet. Note that ePtsHlcrr04 has the highest expression of galETKM and mgIBAC. B) Schematic of the galP operon. The galP SNP occurred at the GalR and GalS transcription factor binding sites. Note that ePtsHIcrr02 has the highest expression of galP. C) Network schematic of CRP-cAMP, GalR, and GalS transcription factor regulation. D) Mutation frequency, metabolite concentration of cAMP, and expression levels of crp, galR, galS, and genes composing the D-galactose symporter and D-galactose $A B C$ transporter. 
Fig. 7 Mutations that alter regulation of the nAcGam import and catabolism. A) schematic of the nagBAC and nagE operons. B) Crystal structure of NagC. NagC is a transcription factor that inhibits expression of nagBAC and nagE operons. Location of the nagC SNP is highlighted in red. The H-T-H DNA-binding domain is highlighted in cyan. C) Mutation frequency and expression levels of genes encoding nAcGam catabolic enzymes (nagBAC) and nAcGam PTS-specific component (nagE). Note that ePtsHlcrr04 has the highest expression of nagBAC and nagE. 
Fig. 8 yrfF and rcs synergistic inactivation. A) Schematic of the interactions of rcsF, yrfF, $r c s C, r c s D$, and rcsB that compose the Rcs phosphorelay system ${ }^{56}$. The Rcs phosphorelay system responds to outer member $(\mathrm{OM})$ and peptidoglycan-related stress in enterobacteria ${ }^{50-53}$, and controls genes related to motility, biofilm formation, virulence, $\mathrm{pH}$, osmolarity, periplasmic quality control, cell division, and DNA replication ${ }^{53-55}$. RcsF is an OM lipoprotein that senses envelope stress and activates the signalling cascade via interaction with YrfF. Under non OM or peptidoglycan-related stress, RcsF is spatially segregated from other Rcs components by BamA, a component of the beta-barrel assembly machinery, which funnels RcsF through the Beta-barrel OmpA. When the cell wall is damaged, RcsF is no longer sequestered by BamA, and RcsF interacts with inner membrane (IM) bound YrfF. YrfF constitutively inhibits the autophosphorylation of RcsC and subsequent phosphorylation of RcsD by RcsC. RcsF interaction with YrfF prevents this inhibition allowing RcsD to phosphorylate and activate RcsB and RcsA, 
which activate downstream target genes. B) yrfF is an essential gene in $E$. coli ${ }^{65}$, and inhibits activation of the Rcs phosphorelay system $^{56}$. Overactivation of the Rcs phosphorelay system leads to acid intolerance. In addition, a yrfF mutant is only viable when combined with deletions in $\operatorname{rcs} B, r \operatorname{cs} C$, and $\operatorname{rcs} D$ that inactivate the RCS system ${ }^{56}$. B) Mutations in yrfF in ePtsHlcrr01 and 03 that were most likely deleterious. The frameshift deletion in yrfF in ePtsHlcrr01 most likely led to a peptide aggregate, and the SNP in ePtsHIcrro3 led to a truncated peptide. The mobile element insertion (MOB) in yrfF in ePtsHlcrrO2 and 04 appeared to silence gene expression. the $\mathrm{MOB}$ insertion in $\operatorname{rcs} A$ in ePtsHlcrr02 led to a nonsense mutation. The MOB insertion in rcs $D$ in ePtsHlcrrO4 led to a nonsense mutation. C) Crystal structure of RcsB ${ }^{66}$. SNPs in rcsB ePtsHlcrr01 targeted the receiver and the H-T-H LuxR-type DNA binding domain ${ }^{53}$ (T195A) and the response regulator receiver domain $(\mathrm{Y} 73 \mathrm{H})$. The SNP in rcsB ePtsHlcrr03 targeted the H-T-H LuxR-type DNA binding domain $^{53}$. Mutations are highlighted in red. D) Mutation 
frequency and gene expression of $y r f F$, and Rcs phosphorelay components. 
Fig. 9 Knockout of ptsHlcrr induced a lexA-mediated SOS response ${ }^{57}$ (Panels A-C). A) Under normal physiological conditions, LexA represses the expression of its own operon, recA, and SOS responder operons. B) When DNA is damaged, single stranded DNA (ssDNA) binds to recA subunits to form a protease. This protease cleaves LexA, leading to a decrease in LexA repression, and expression of SOS responder operons. C) Expression profiles of $\operatorname{rec} A$, lexA, and SOS response genes that are regulated by LexA. The lexA-mediated SOS response manifested into a large chromosomal duplication event that resulted in an increased gene dosage of ATP synthase complex genes that most likely aided in restoring the energy charge (Panels D-F). D) Reads vs. genome position. Inset highlights the duplicated region near 4MB. E) Schematic of the ATP synthase operon genes. F) Expression levels of the ATP synthase complex genes. ePtsHlcrr02/04 ATP synthase genes are significantly elevated; note that the energy charge for ePtsHIcrro2/04 is not significantly different than Ref. 


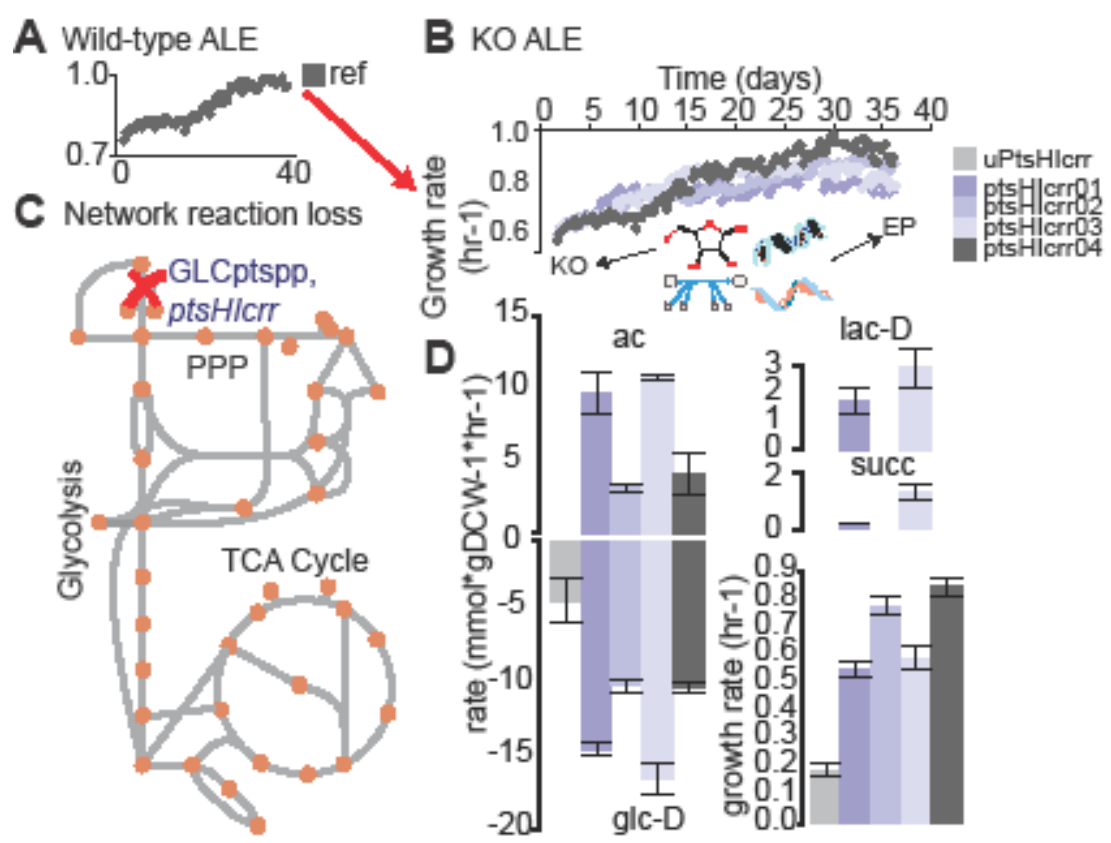



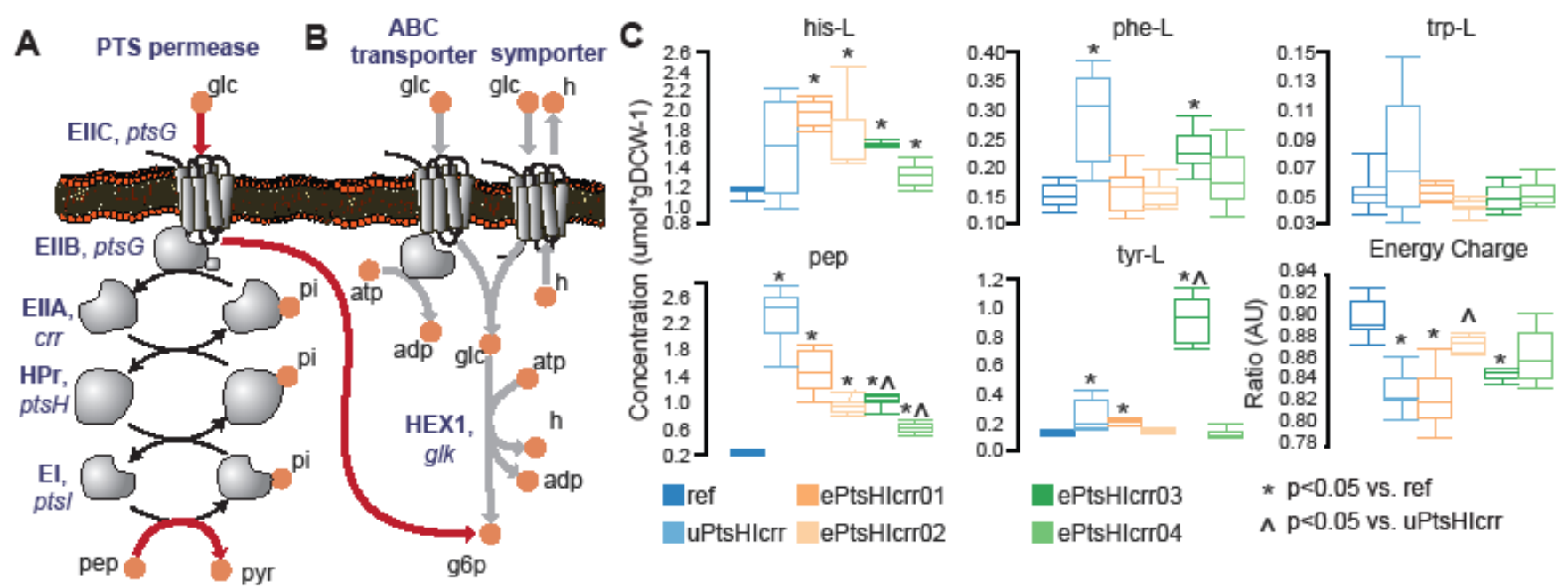


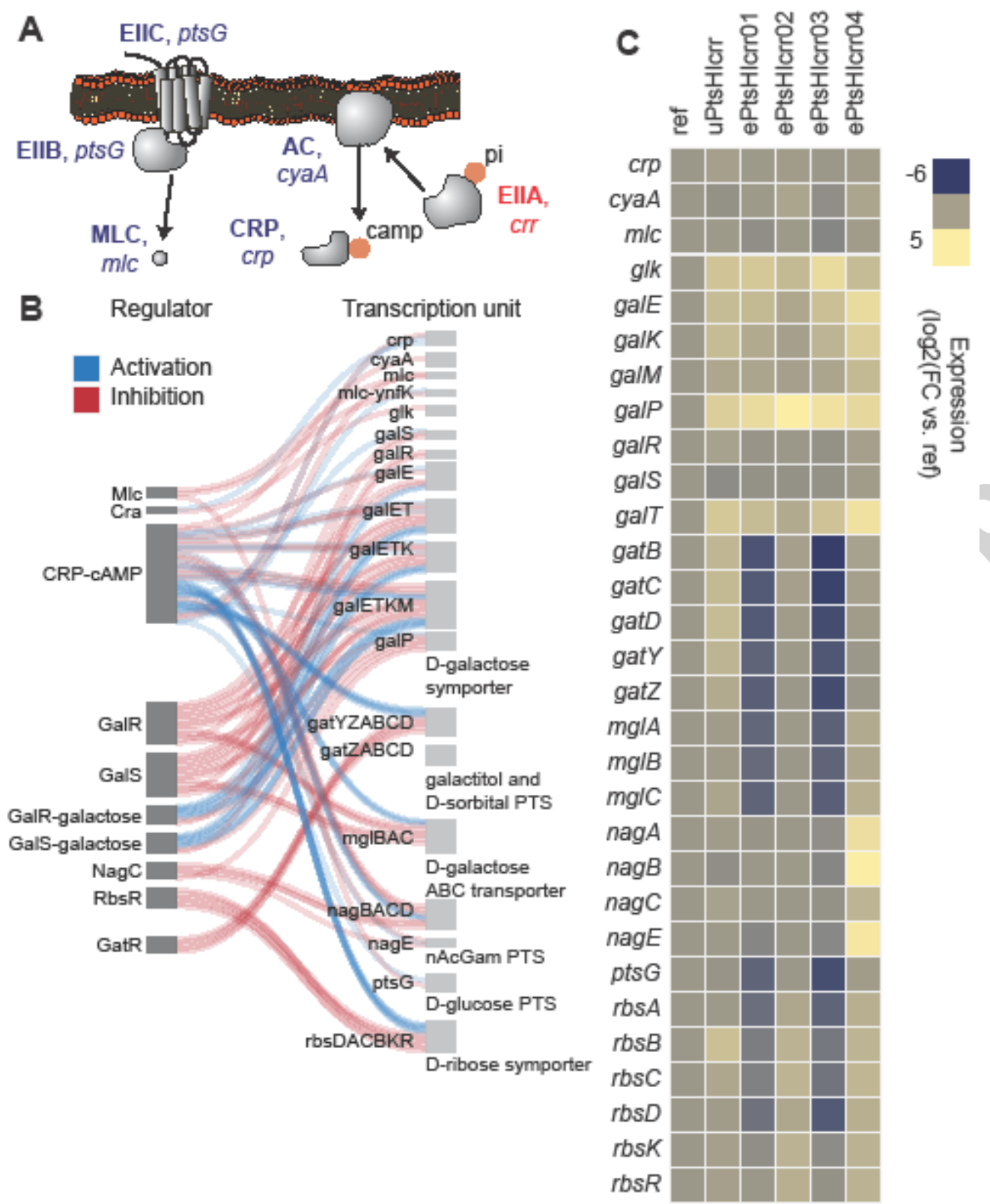



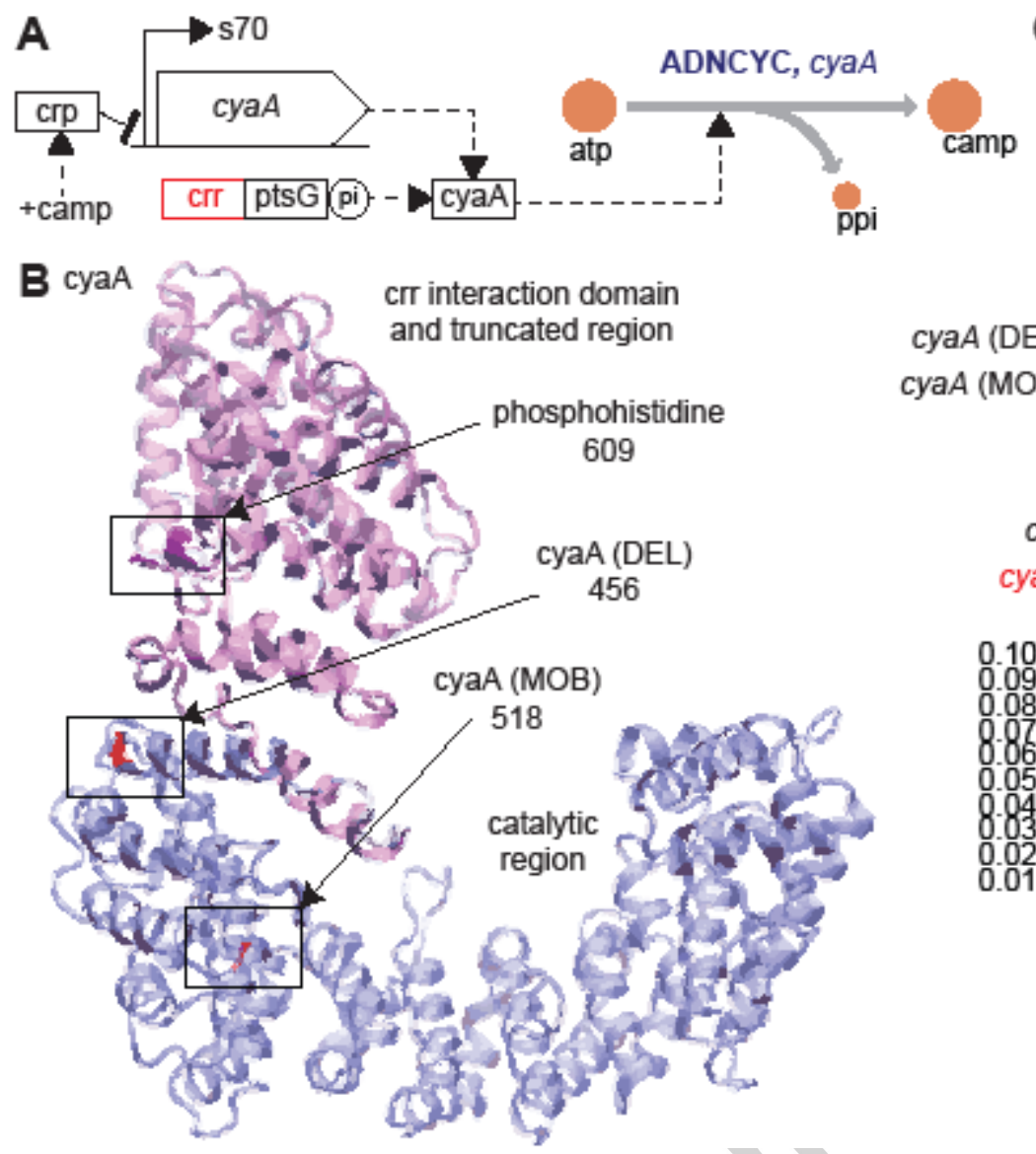
cyaA (DEL) cyaA (MOB)
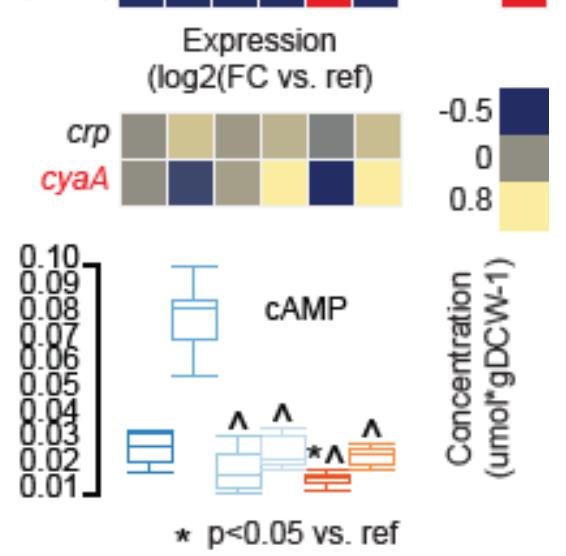

$\wedge p<0.05$ vs. ptsHIcrr
A

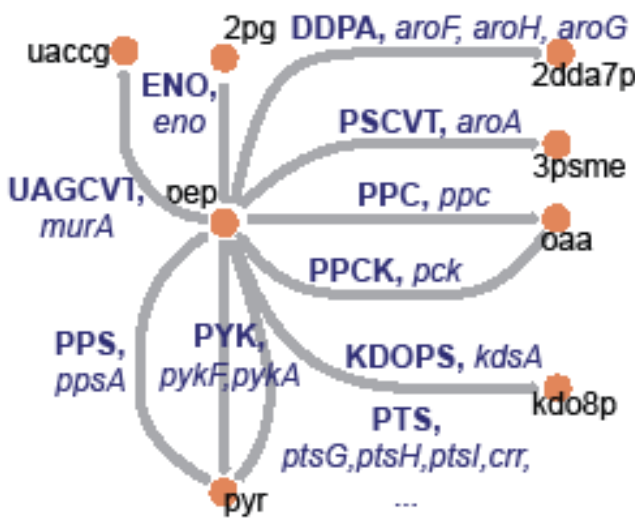

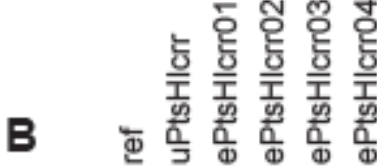

Flux (mmol* $\mathrm{gDCW}-1^{\text {*hr- }}$ )
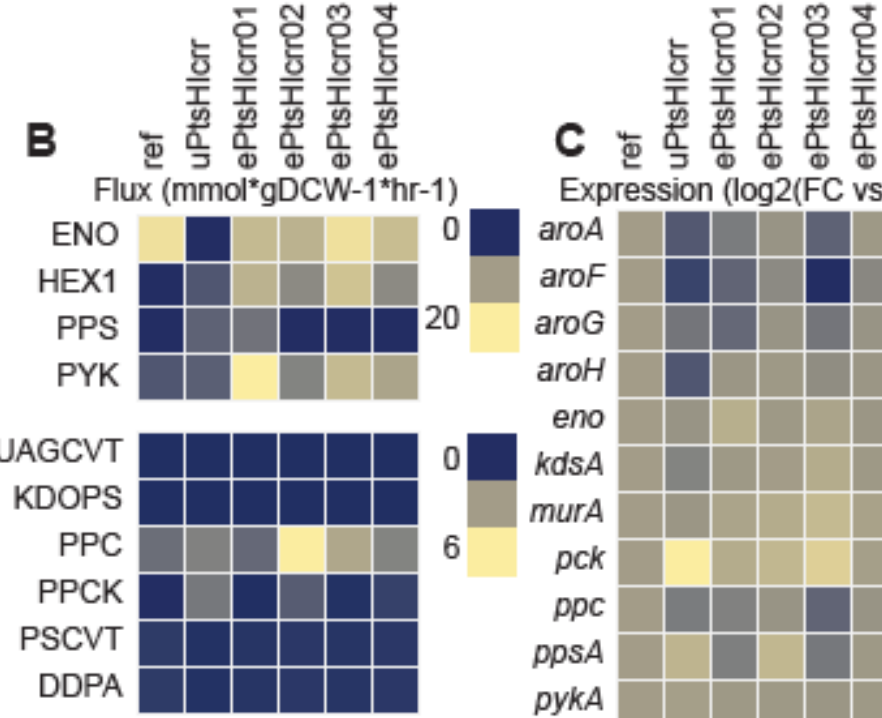
Expression (log2(FC vs. ref))

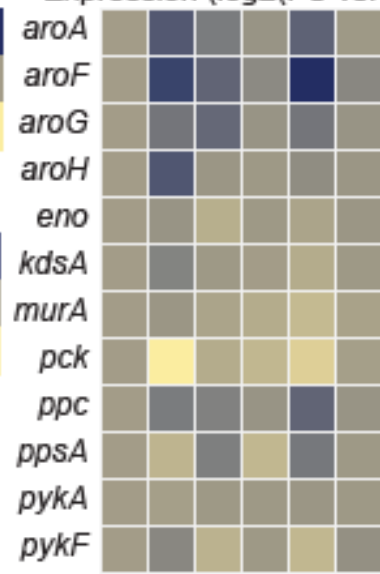


A

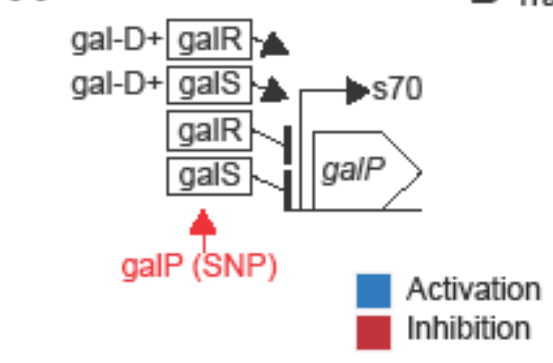

C

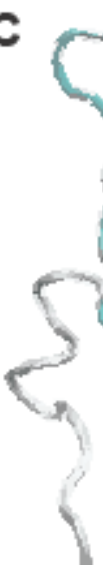

galR (SNP) S328A
B Transcription Factor Transcription unit

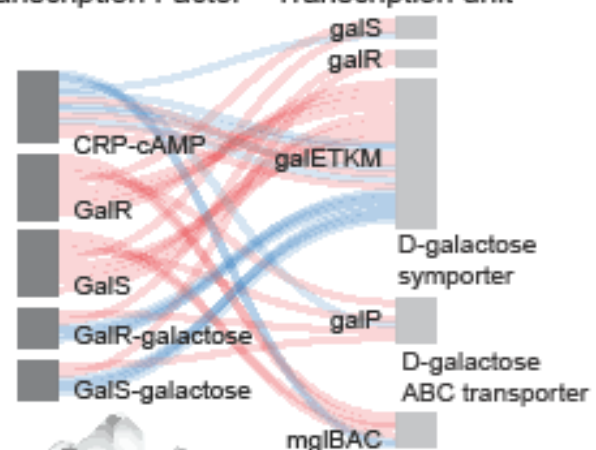

mgIBAC

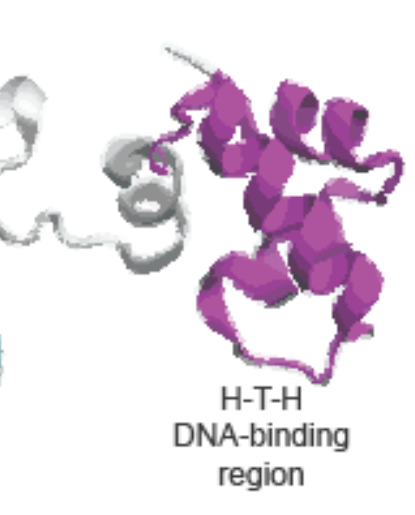

small molecule

binding domain
D
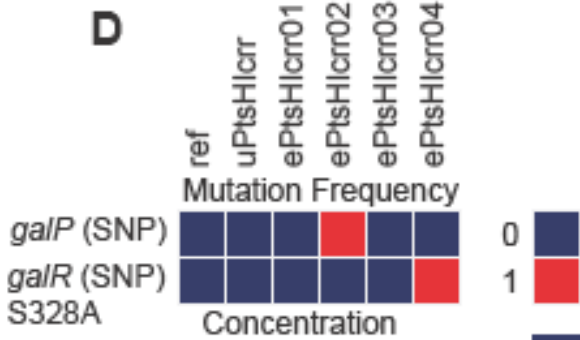

$\mathrm{S} 328 \mathrm{~A}$

(umol'gDCW-1 normalized) -3.5

camp

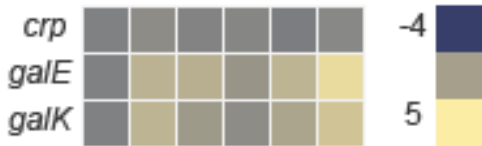

galm

galP

galR

gals

galT

$g / k$

$m g l A$

$m g l B$

$\mathrm{mg} / \mathrm{C}$

$\sqrt{0+7}$

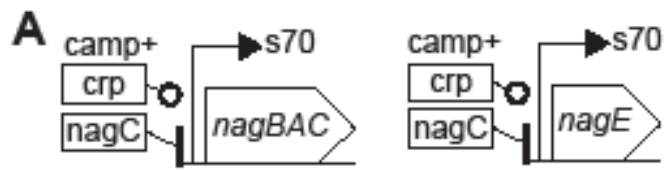

B

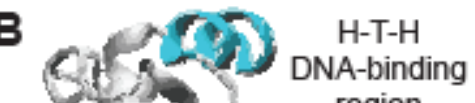

5 region

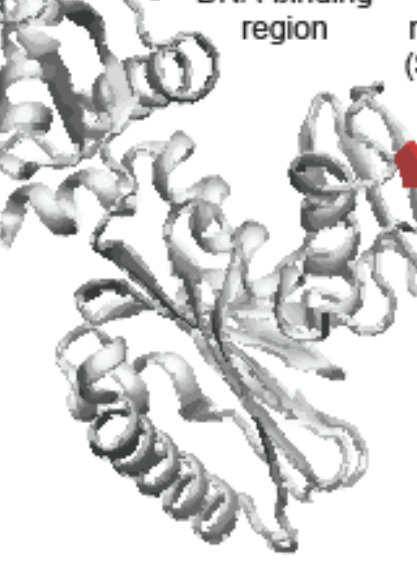

\section{nagC} (SNP)

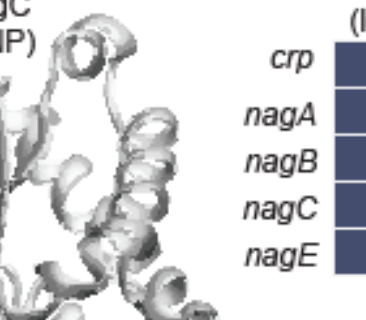



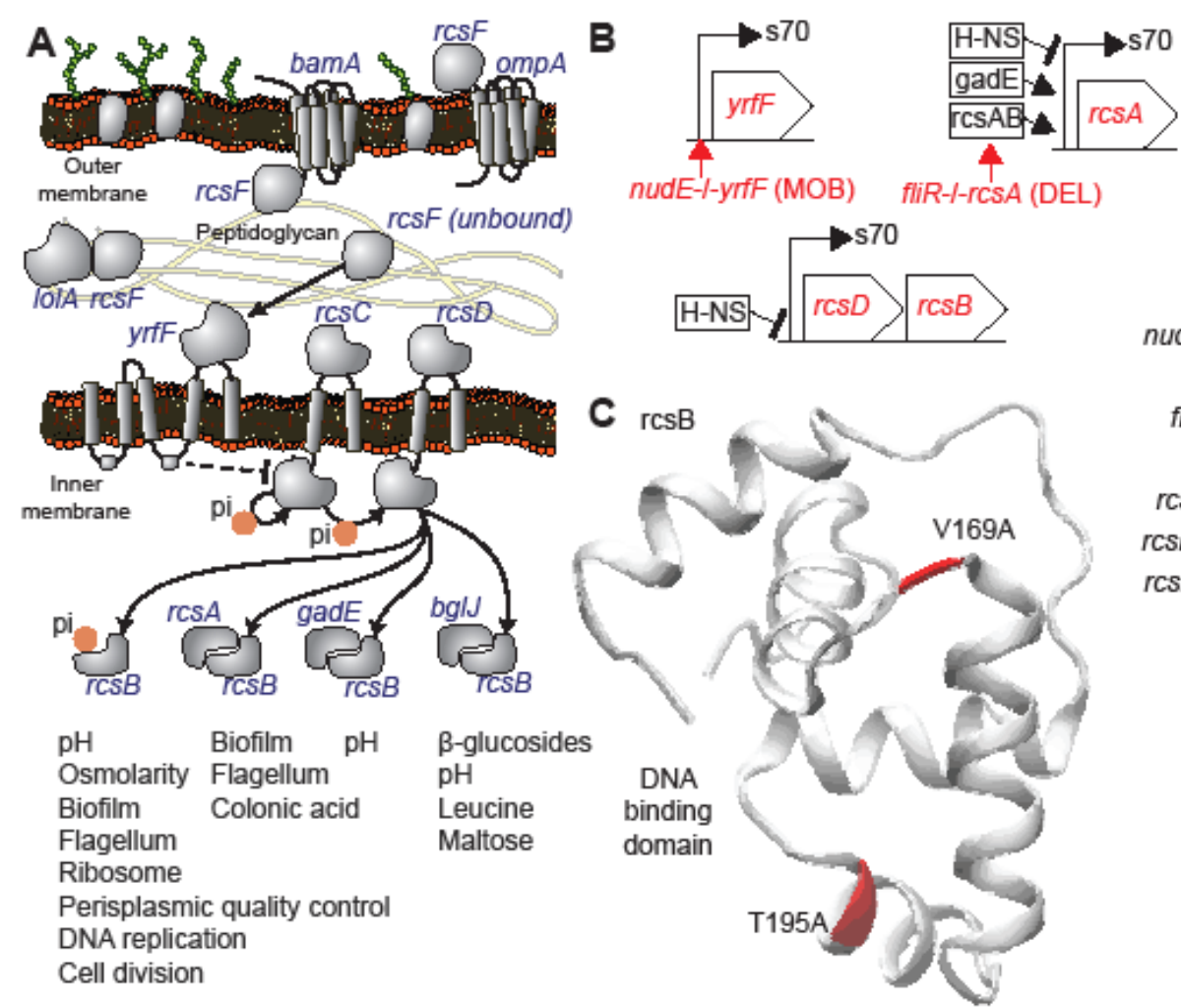

D

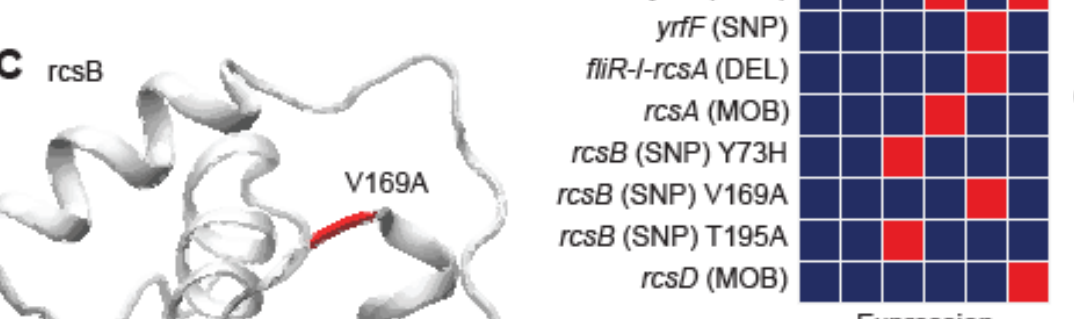

0

Expression (log2(FC vs. ref))

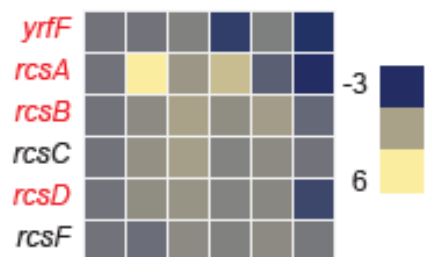


A No DNA damage

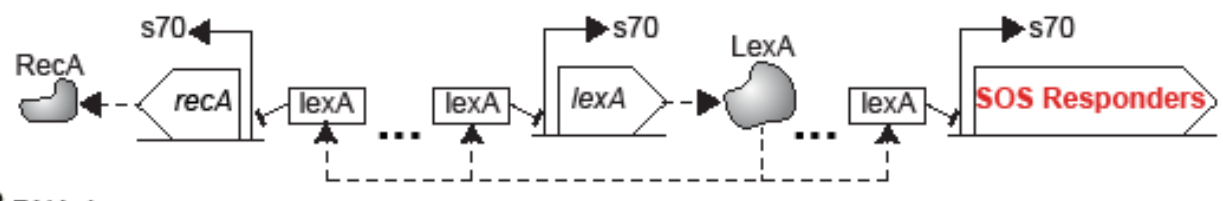

B DNA damage
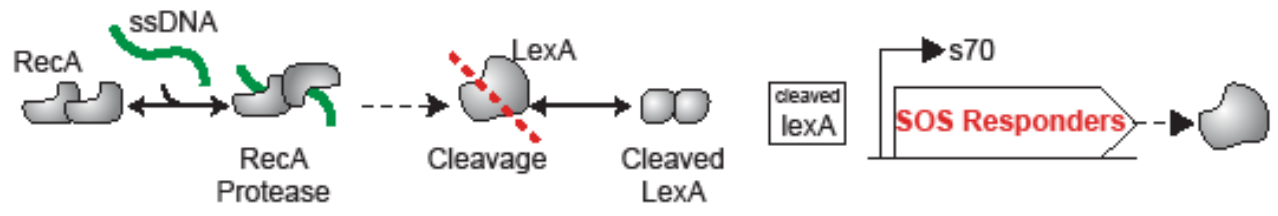

$$
\text { LexA }
$$

D

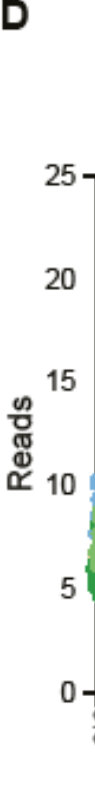

\section{0}

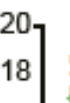

16

14

12

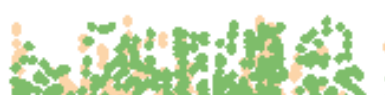

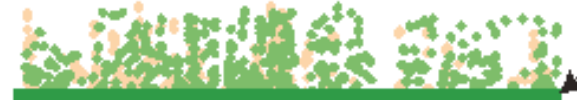

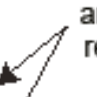

amplification

reads mean $\quad$ uPtsHIcrr ePtsHIcrr01 ePtsHIcrr02

ePtsHIcrr03 aePtsHIcrr04
C
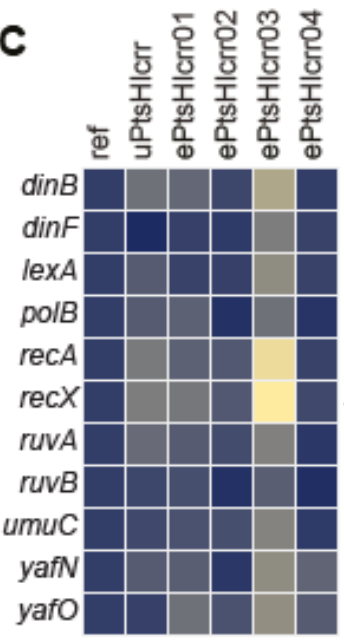

Expression $(\log 2(\mathrm{FC}$ v. ref')

E

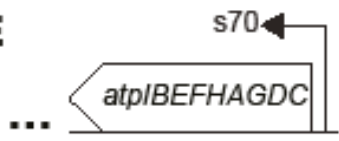

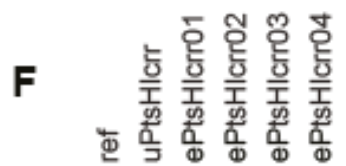

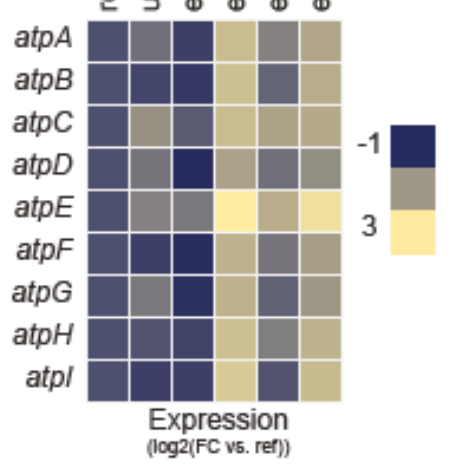

\section{Highlights:}

- PTS Knockout-out in E. coli and adaptive laboratory evolution (ALE) 
- Energy deficiency overcome by high fermentation or respiration.

- Synergist mutations deactivated the Rcs phosphorelay system aided recovery of fitness

- High intracellular aromatic metabolite levels found in evolved strains. 